\title{
Maxillary Protraction in an Adolescent Female Patient: A Case Report
}

\author{
${ }^{1}$ Department of Orthodontics and Dentofacial Orthopedics, Modern \\ Dental College and Research Center, Indore, Madhya Pradesh, India \\ 2Department of Oral and Maxillofacial Pathology, Modern Dental \\ College and Research Center, Indore, Madhya Pradesh, India \\ ${ }^{3}$ Department of Oral Pathology \& Microbiology, Dr. D. Y. Patil \\ Dental College \& Hospital, Dr. D. Y. Patil Vidyapeeth, Pune, \\ Maharashtra, India \\ ${ }^{4}$ Department of Orthodontics, C.S.M.S.S. Dental College and \\ Hospital, Kanchanwadi, Aurangabad, Maharashtra, India \\ ${ }^{5}$ Department of Oral Medicine and Radiology, Index Institute of \\ Dental Sciences, Indore, Madhya Pradesh, India
}

Suruchi Jatol-Tekade ${ }^{1}$ Satyajit Ashok Tekade ${ }^{2}$ Sachin C. Sarode ${ }^{3}$ Vishal Patni ${ }^{4}$ Vihang Naphde

\begin{abstract}
Address for correspondence Suruchi Jatol-Tekade, BDS, MDS, Department of Orthodontics and Dentofacial Orthopedics, Modern Dental College and Research Center, Gandhi Nagar, Airport Road, Indore 453112, MP, India (e-mail: suruchitekade@gmail.com).
\end{abstract}

Dent J Adv Stud 2018;6:118-121

\begin{abstract}
Keywords

- growth modulation

- class III malocclusion

- tandem appliance

Tandem appliance is preferred over face mask mainly because of compliance reasons. In the given case report, clinicians have used tandem appliance for correcting skeletal class III malocclusion patient in later stage of growth. Operators utilized residual growth. Facial harmony was achieved by gaining positive overjet. Adolescent class III malocclusion is challenging to treat. Occurrence of class III malocclusion is just $5 \%$ in India. If patient reports in growing stage, clinicians get many relevant treatment options. Lack of knowledge about growth modulation therapy causes loss of growing stage of patients. This situation reduces available treatment modalities. Here is a case of female patient with class III malocclusion who is near completion of her growth; maxillary growth was stimulated in forward direction taking anchorage from overgrown mandible with the help of tandem appliance.
\end{abstract}

\section{Introduction}

Growth modulation therapy is shaping and guiding growing jaw bone to achieve appropriate occlusion and suitable facial proportion. Proper use of growth potential creates desirable changes in the development of dentoalveolar structures. Avoiding growing malocclusion and accelerating proper growth are the general guidelines of dentofacial orthopaedic treatment. So, it is necessary to catch the growth at appropriate time and utilize whatever growth potential is remaining. Class III malocclusions are that way difficult to treat by growth modulation. As maxillary growth stops early according to cephalocaudal index and before the parents notice developmental problem. The treatment results rely on left overgrowth potential in class III patients. ${ }^{1}$ Thorough knowledge of craniofacial growth in all the three directions is must to determine treatment time and success. According to Krogman, maxillary bone stops growing in width, then in length, and then in height. ${ }^{2}$ But recent studies by Yavuz et al and Chen et al show that though "growth magnitude" has reduced maxillary bone growth in height till puberty, its growth rate may vary in girls than boys in terms of early cessation of growth.,4

Dentofacial deformities are best treated in growing age to achieve proper facial profile and function. The early orthopaedic intervention of class III deformity where maxillary growth is deficient guides the orofacial growth and development, giving facial muscular balance. ${ }^{5}$ Early treatment allows greater opportunity to modify craniofacial skeletal growth, improved confidence of the child, parental satisfaction, and prevents surgical intervention required later thereby reducing the possibility of iatrogenic tooth damage such as paresthesia, root resorption, decalcification, and trauma. ${ }^{6}$ All of this is possible only if patient reports before completion of maxillary growth. Reports are there in the literature utilizing received

July 30, 2018

accepted after revision

September 24, 2018

published online

November 2, 2018
DOI https://doi.org/

10.1055/s-0038-1675250

ISSN 2321-1482.
(C)2018 Bhojia Dental College and Hospital affiliated to Himachal Pradesh University
License terms

() (1) $\odot \circledast$ 
residual growth of maxilla in later stages of growth. ${ }^{7}$ This shows extending our vision and trying to utilize whatever growth is remaining in skeletal malocclusion patients. In addition, other advantages of growth modulation are more over surgical treatment, so clinician should take the chance to improve facial esthetics and occlusion.

\section{Case Report}

A 12-year-old girl with a chief complaint of crowding in upper front teeth came to our clinic. Clinical examination revealed midfacial deficiency and straight profile with anterior divergence. Intraoral examination showed Angle's class III molar relationship, anterior crossbite, upper and lower right second premolars, and left canines were in crossbite ( $\boldsymbol{- F i g}$. 1). There was no occlusal interference or deviation on mandibular closure. The panoramic radiograph showed no missing teeth or pathologies. Cephalometric analysis proved a skeletal class III maxilla-mandibular relationship due to short maxillary length, along with vertical growth pattern, lower incisors found upright over mandibular base. Though upper incisors tried to compensate short maxilla naturally by proclination, anterior crossbite was unavoidable due to overgrown mandible. So not only maxilla was deficient in this case, mandible was also at fault creating skeletal class III. Patient and her parents were made aware of the condition and options available for treating her case. Rather than waiting till adulthood and undergoing surgery, they opted for conservative way.

The upper and lower first permanent molars were banded. A modified tandem appliance (with hyrax expansion screw) was fabricated and cemented ( - Fig. 2). Both the upper and lower arches were bonded with 0.022-inch MBT appliance (3M UNITEK Victory Series). The anterior crossbite bite was corrected after 10 months of active treatment. The profile also showed a significant improvement ( - Fig. 3 ).
The appliance was removed total of 20 months after active treatment phase. Cephalometric evaluation and superimposition revealed skeletal improvement.

\section{Treatment Objectives}

Patient's cephalometric analysis confirmed class III skeletal pattern. The treatment objective was to achieve class I pattern utilizing left overgrowth potential with the help of maxillary protraction appliance. Patient was having crossbite, and second objective was to achieve positive overjet and overbite, which will serve as a lock for growing mandible. Third objective was to relieve single tooth crossbite in posteriors. Achieving pleasant soft tissue profile was clinician's aesthetic goal. Retention phase is decisive in growing class III patients. Measures to prevent relapse were taken as chin cap wear for at least 1 year.

\section{Treatment Plan}

Orthopaedic treatment was planned to stimulate remaining growth potential of maxilla. The face mask could have been planned but it requires lot of patient compliance. Tandem appliance, taking anchorage from mandible and protracting maxilla, was preferred over face mask. Hence, rapid maxillary expansion was done to loosen the maxilla from circum-maxillary sutures ${ }^{8,9}$ and sagittal maxillary advancement with tandem appliance to correct class III skeletal malocclusion was tried. Patient's arches were square shaped. To reduce lower arch crowding and to get positive overjet, lower left first premolar was extracted. It helped to relieve crowding and to align the arch. Fixed orthodontic mechanotherapy with preadjusted edgewise appliance was performed for the final detailing of occlusion.

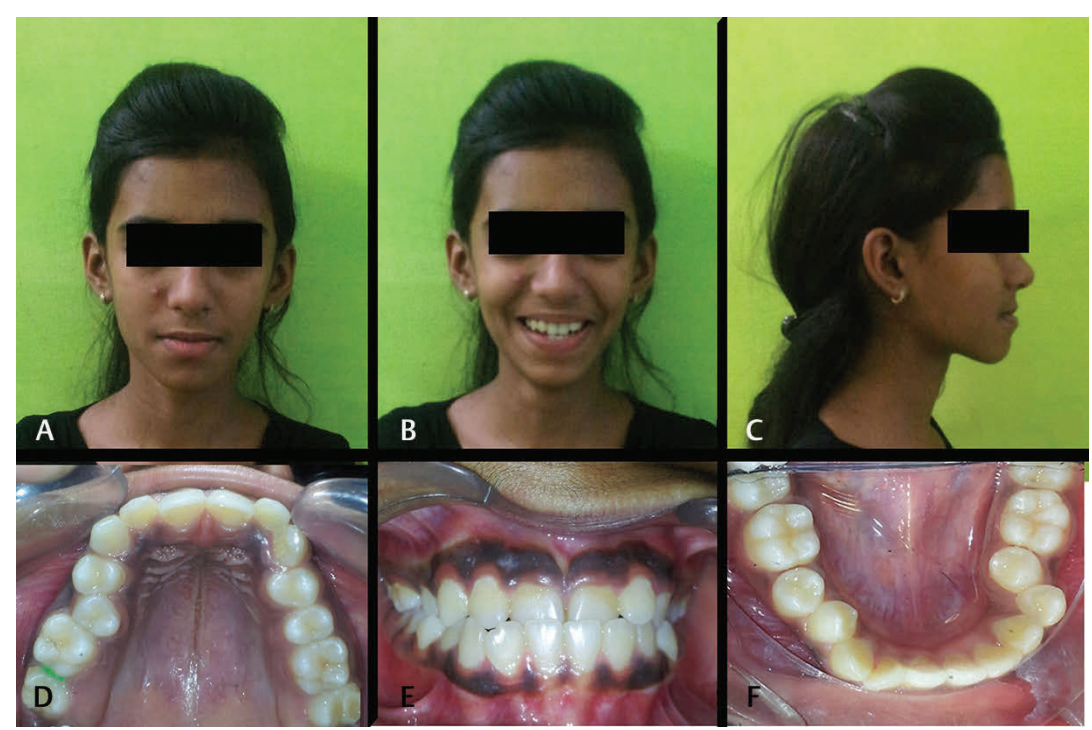

Fig. 1 Pretreatment photographs: (A) Extraoral front, (B) extraoral smiling, (C) extraoral profile, (D) occlusal view of maxillary arch, (E) intraoral in occlusion front, and (F) occlusal view of mandibular arch. 


\section{Treatment Progress}

The patient was treated with tandem appliance along with fixed orthodontic appliance. The upper assembly comprised a hyrax expansion screw with first molars and first premolars banded. The protraction hooks in the maxilla were placed distal to the canines, in the premolar region so that the elastic force passes exactly through the center of resistance of the maxilla. Care was taken for no rotation of occlusal or palatal plane. On both sides, a force of 400 to $450 \mathrm{~g}$ was applied bilaterally for 14 to 16 hours per day with the help of extraoral elastics. Leveling and aligning followed by space closure was done in the lower left first premolar extraction region. For correcting the anterior crossbite, posterior bite blocks were given to open the reverse jet and let the maxillary teeth come forward with protraction pull. Blocks were reduced eventually. Finishing and detailing were achieved before debonding.

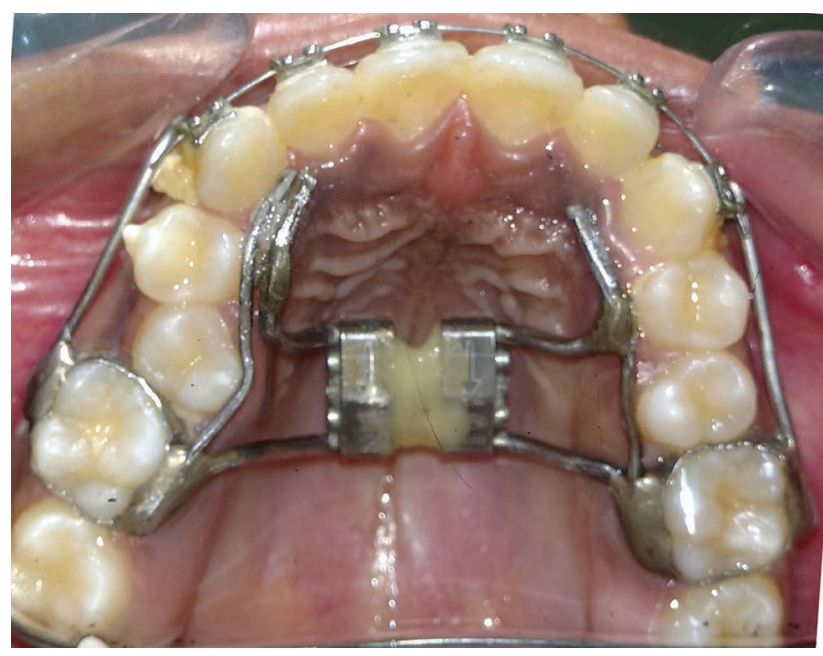

Fig. 2 Tandem appliance in place, showing hyrax banded on upper arch palatally and hooks to engage protraction elastics are visible buccally.
Full expression of tip and torque was achieved on $0.021 \mathrm{X}$ 0.025-inch stainless steel wire.

\section{Treatment Results}

The patient was assessed every month till the satisfactory results achieved. Progress records were taken after 20 months of active treatment. Favorable position of maxilla and normal overjet overbite was achieved.

Postprotraction cephalometric tracings revealed a forward movement of maxilla. The ANB angle increased from 3 to 0 degree. Point $N$ perpendicular to $(\perp)$ point $A$ increased by $1 \mathrm{~mm}$. Upper incisors were proclined. The point A also shifted anteriorly by $1 \mathrm{~mm}$ approximately. Patient was advised to wear chin cap for retention of the achieved results for another 6 months. The patient is on regular follow-up.

\section{Discussion}

Orthopaedic appliances play vital role in the correction of jaw deformities in orthodontics. The effectiveness of these growth modifications appliances depends on skeletal maturity. ${ }^{4}$ Otherwise orthognathic surgery is the treatment option to correct jaw deformities after growth cessation. Dentofacial orthopaedic treatment should prevent undesirable growth-related changes and should allow desirable ones. Findings of Chen et al showed that maxillary protraction was more effective when started at an early stage. ${ }^{4}$ Yavuz et al found that incremental growth changes were greater in 10 to 12 years old female patients than in 12 to 14 years old female patients. ${ }^{3}$ In the given case, clinicians have taken advantage of remaining growth in a patient with maxillary discrepancy. Patient was a class III subject with maxillary deficiency in arch width and anteroposterior length. Though her maxillary growth is on the verge of completion, preventing overgrowth of mandible was necessary. Best retention for holding mandibular growth

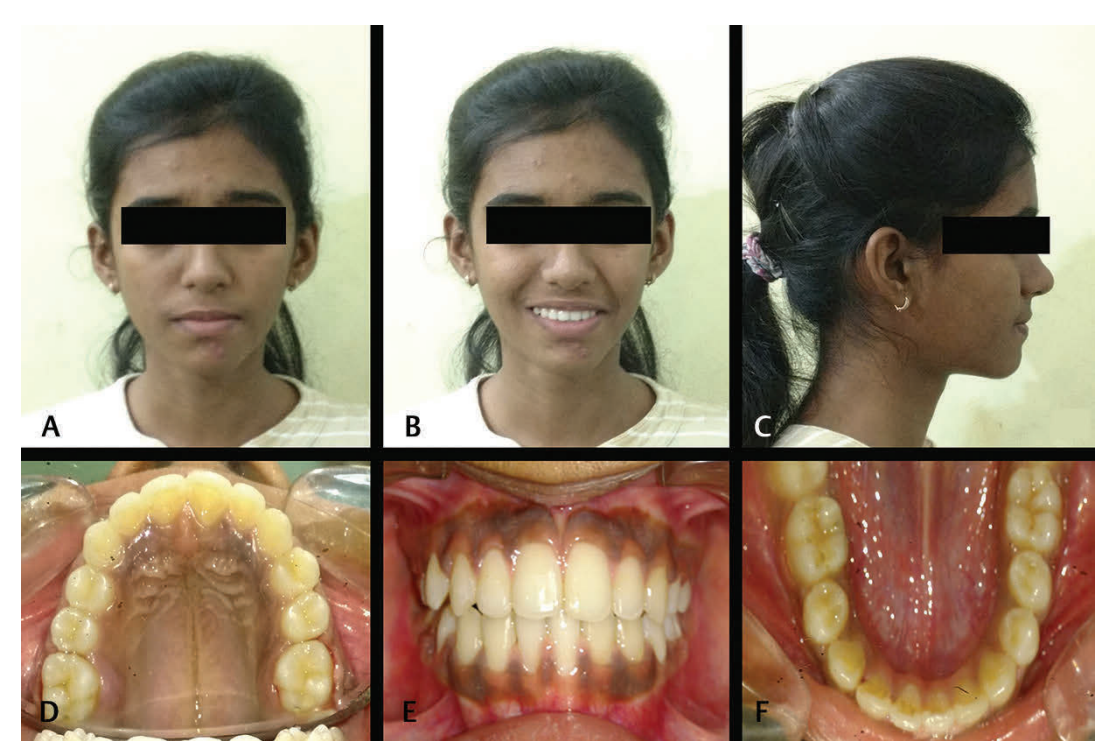

Fig. 3 Post-treatment photographs: (A) Extraoral front, (B) extraoral smiling, (C) extraoral profile, (D) occlusal view of maxillary arch, (E) intraoral in occlusion front, and (F) occlusal view of mandibular arch. 
is positive overjet and chin cap. Rapid maxillary expansion with protraction of maxilla with tandem appliance was considered as one of the best treatment options considering patient compliance and treatment results. Direction of protraction force was carefully monitored as patient was vertical grower and clockwise rotation of mandible was prevented. As per the research data of Jamison et al, sagittal relationship of maxilla and mandible changed from 8 to 17 years of age. ${ }^{10}$ Same results were shown by Sherman et al in relation to wits appraisal, in the age of 4 to 24 years. ${ }^{11}$ Kluemper and Spalding $^{12}$ enlightened us about orthopaedic treatment timing of older children. Skeletal class III patients with a maxillary deficiency get considerable results from early orthopaedic treatment. Yet, growth modulation may produce more "favorable" changes for older children and adolescents than thought in the past. For this small "favorable" change, chance should be taken to avoid surgical intervention in future. And in this case report, results prove worthiness of trying maxillary protraction in adolescent female patient. Orthopaedic correction of the mild-to-moderate skeletal class III malocclusion should also include regular follow-up and growth evaluation.

Although class III cases are barely reported at early age in India, awareness about their treatment options and consequences of no treatment is necessary.

\section{Conclusion}

The basis of all options available for treating developing class III malocclusion is to improve the facial profile and to allow the normal growth of the craniofacial bones. Earlier the reporting of class III malocclusion, sooner will be the diagnosis, and simpler and faster treatment! So even if a child in late puberty comes with class III malocclusion, clinician should try bone modulation approaches to give proper craniofacial harmony.

\section{Conflict of Interest}

None declared.

\section{References}

1 Sugawara J, Asano T, Endo N, Mitani H. Long-term effects of chincap therapy on skeletal profile in mandibular prognathism. Am J Orthod Dentofacial Orthop 1990;98(2):127-133

2 Krogman WM. Craniofacial growth, prenatal and postnatal. In: Cooper HK, Harding RL, Krogman WM, Mazaheri M, Millard RT, eds. Cleft Palate and Cleft Lip: A Team Approach to Clinical Management and Rehabilitation. Philadelphia: W.B. Saunders; 1979:22-107

3 Yavuz I, Ikbal A, Baydaş B, Ceylan I. Longitudinal posteroanterior changes in transverse and vertical craniofacial structures between 10 and 14 years of age. Angle Orthod 2004;74(5):624-629

4 Chen L, Liu J, Xu T, Lin J. Longitudinal study of relative growth rates of the maxilla and the mandible according to quantitative cervical vertebral maturation. Am J Orthod Dentofacial Orthop 2010;137(6):736.e1-736.e8, discussion 736-737

5 Almeida MR, Almeida RR, Oltramari-Navarro PV, Conti AC, Navarro RdeL, Camacho JG. Early treatment of Class III malocclusion: 10-year clinical follow-up. J Appl Oral Sci 2011;19(4):431-439

6 Bishara SE, Justus R, Graber TM. Proceedings of the workshop discussion on early treatment. Am J Orthod Dentofacial Orthop 1998;113:5-6

7 Jatol-Tekade S, Tekade SA, Sarode SC, Sarode GS, Patil S. Treatment of Class III malocclusion at later stage of growth. World J Dent 2017;8(5):1-5

8 McNamara JA Jr. An orthopedic approach to the treatment of Class III malocclusion in young patients. J Clin Orthod 1987;21(9):598-608

9 Turley PK. Orthopedic correction of Class III malocclusion with palatal expansion and custom protraction headgear. J Clin Orthod 1988;22(5):314-325

10 Jamison JE, Bishara SE, Peterson LC, DeKock WH, Kremenak CR. Longitudinal changes in the maxilla and the maxillary-mandibular relationship between 8 and 17 years of age. Am J Orthod 1982;82(3):217-230

11 Sherman SL, Woods M, Nanda RS, Currier GF. The longitudinal effects of growth on the Wits appraisal. Am J Orthod Dentofacial Orthop 1988;93(5):429-436

12 Kluemper GT, Spalding PM. Realities of craniofacial growth modification. Atlas Oral Maxillofac Surg Clin North Am 2001;9(1):23-51 